\title{
Produção de aves em sistema orgânico
}

$\underline{\text { Gessica de Souza Azevedo }}^{1}$; $\underline{\text { Joana Patrícia Lira de Souza }}^{2} ;$ José Avelino Cardoso $^{3}$; Pedro $\underline{\text { Henrique Haddad Araujo }^{4}}$; Ernestina Ribeiro dos Santos Neta ${ }^{5}$ Michele Pereira Vilas Novas ${ }^{6}$

${ }^{I}$ Aluna de Graduação em Zootecnia pela Universidade Federal Rural da Amazônia-UFRA, Campus de Parauapebas - Pará.

${ }^{2}$ Prof $^{a}$ Dr $^{a}$ Ciência Animal Tropical pela Universidade Federal do Tocantins-UFT, Campus de Araguaína.

${ }^{3}$ Prof $^{\circ}$. MS. Em Agronomia pela Universidade de Brasília.

${ }^{4}$ Aluno de Graduação em Engenharia Agronômica pelo Instituto Federal de tecnologia, Educação e Ciência de Tocantins-IFTO, Campus Dianópolis.

${ }^{5}$ Aluno de Graduação em Engenharia Agronômica pelo Instituto Federal de tecnologia, Educação e Ciência de Tocantins-IFTO, Campus Dianópolis.

${ }^{6}$ Prof ${ }^{a}$ de Língua Portuguesa do Instituto Federal de Educação, Ciência e Tecnologia do Estado do Tocantins IFTO, Campus Dianópolis.

*Autor para correspondência, E-mail:gessicaazevedo1@hotmail.com

RESUMO. O mercado consumidor está cada vez mais preocupado com os alimentos de origem animal que consomem e principalmente nas condições em que os mesmos são mantidos durante o período de produção. A avicultura de postura é um dos setores em que essas preocupações tornam-se cada vez mais exigentes, pois, na avicultura industrial esses animais são criados em gaiolas durante todo o ciclo de produção, apesar de o espaço ser considerado suficiente para que essas aves possam movimentar-se, tal espaço não permite que as aves possam expressar algumas características do seu comportamento natural (tomar banho de areia, subir e descer de poleiros, terem um ambiente amplo para explorar) esse comportamento é observado em sistema semi confinado, caipira, ou orgânico. Apesar de esses sistemas terem certa semelhança, se diferem em alguns aspectos, o orgânico é certificado por órgãos responsáveis, que podem ser empresas certificadoras, que por sua vez são fiscalizadas pelo Ministério da Agricultura e Abastecimento, o que o diferencia dos demais. A sanidade no caso da criação orgânica deve ser feita sem o uso que medicamentos veterinários, e apenas as vacinas de caráter obrigatório devem ser oferecidas aos animais, garantindo uma produção dentro dos padrões de exigência da organização internacional de produção orgânica.

Palavras chaves: Avicultura, orgânico, sanidade

\section{Poultry production in organic system}

ABSTRACT. The consumer market is increasingly concerned with animal foods they consume and especially the conditions in which they are kept during the production period. The posture of poultry is one of the sectors in which these concerns become increasingly demanding, because in industrial poultry farming these animals are kept in cages throughout the production cycle, although the space is considered sufficient for these birds can jogging, that space does not allow the birds to express some characteristics of their natural behavior (taking sand bath up and down perches, have ample room to explore) this behavior is observed in semi-confined system, hillbilly, or organic. Although these systems have certain similarities, they differ in some respects, the organic is certified by bodies, which can be certifying companies, which are in turn supervised by Ministry of Agriculture and Supply, which differs from other . Sanity in the case of organic creation must be made without the use of veterinary medicinal products, and only the mandatory vaccine should be offered to animals, ensuring production within the requirement of the international standards of organic production organization.

Keywords: Poultry, organic, health 


\section{Introdução}

A avicultura tem apresentado investimentos tecnológicos visando melhorar o desempenho produtivo das aves, possibilitando avanços na produção por meio de técnicas de melhoramento genético, alimentação, manejo e sanidade (Furlan et al., 2006). As linhagens de poedeiras atuais são diferentes das antigas, quanto ao temperamento, potencial produtivo, consumo de ração, ganhos de peso, viabilidade e tipo de ovos (Menezes et al., 2009). Segundo dados da União Brasileira de Avicultura o Brasil em 2013, produziu 34,12 bilhões de ovos, número 7,4\% maior comparado ao ano de 2012. Já em relação ao consumo per capita, o crescimento chegou a 168,7 unidades per capita, ao ano, contra 162 obtidas no ano anterior.

No entanto, outro cenário que tem ganhado extrema importância desde os anos 90 é a preocupação das autoridades públicas e das organizações não governamentais (ONGs), com o meio ambiente, principalmente no que se refere ao efeito estufa, preservação dos ecossistemas, poluição dos mares e rios e o uso de produtos químicos, como consequência a geração de lixos domésticos e industriais (Arenales et al., 2008). Diante disso, foram propostas mudanças nos sistemas atuais de produção avícola para que possam suprir as exigências do mercado preocupado com as formas de criação, dando lucros satisfatórios aos produtores que adotarem os sistemas de criações Caipiras e Orgânicas.

Em 1972 o termo "agricultura orgânica" foi definido pela Federação Internacional dos Movimentos de Agricultura Orgânica, que após sua criação passou a estabelecer normas para que os produtos fossem comercializados com o selo de comprovação que o manejo realizado era de origem orgânica. Tais normas, além de proibirem os agrotóxicos e restringirem a utilização dos adubos químicos, também incluíam ações de conservação dos recursos naturais e aspectos éticos nas relações sociais e no trato com os animais (Khatounian, 2011).Apesar dessa importância no que se refere à criação de animais, ainda necessita de trabalhos que possam explicar de maneira fisiológica e científica, como ocorre a reação de fitoterápicos utilizados no manejo sanitário.

\section{Avicultura caipira ou colonial}

No sistema colonial as aves são criadas em confinamento, aproximadamente até os 28 dias de vida, depois desse período, passam ater livre acesso, durante o dia, a um piquete ao redor do aviário, onde terão forragem como outra fonte de alimento, além de terem espaço para caminhar livremente. Isso propicia ao animal um maior bem estar, diminuindo o nível de estresse entre eles. Os frangos e ovos coloniais são produzidos obedecendo à legislação federal (Ofício circular DOI/DIPOA no 007/99, O.C. DIPOA N ${ }^{\circ} 60 / 99$ ) (MAPA, 1999).

Devido à diversidade de alimentos oferecidos às aves criadas nesse sistema, e também a oferta de pastagem, a gema acaba possuindo em uma coloração mais amarelada, sendo esta uma característica muito apreciada pelos consumidores. Outro fator é que a ração fornecida para as aves é constituída de produtos de origem vegetal, sendo proibido o uso de aditivos e promotores de crescimento, bem como ingredientes de origem animal (Zabaleta, 2013).

O programa de seleção das aves para serem criadas em sistema caipira, procurou encontrar um ponto de equilíbrio entre o passado e o futuro e entre a rusticidade e a produtividade, apresentando aves com potencial de produção de 270 a 300 ovos ao ano e também aves especializadas para produção de carne com a vantagem da comercialização de um produto diferenciado, com melhor remuneração por parte do mercado consumidor (Pasian, 2007).

\section{Avicultura industrial ou convencional}

O sistema industrial ou convencional é utilizado em granjas para fins de exploração comercial, as linhagens são geneticamente selecionadas considerando a alta taxa de crescimento e excelente eficiência alimentar. Essa criação é de caráter intensivo, seguindo as normas sanitárias vigentes e não possuem restrição quanto ao uso de anticoccidianos, promotores de crescimento, quimioterápicos, e também quanto ao uso de ingredientes de origem animal na dieta (Alvarado Hullanco, 2004). É um sistema voltado para se obter uma maior produção no menor tempo possível. Dessa forma, a nutrição, sanidade, ambiente e manejo devem estar adequados para propiciar a expressão de todo o potencial das poedeiras comerciais (Pasian, 2006).

Embora tais condições da criação industrial tenham proporcionado ganhos econômicos, também têm resultado em problemas quanto ao 
bem-estar das aves, dada a utilização de certas práticas de criação e de manejo (Pasian, 2007).

Assim, a conversão de um sistema de produção animal convencional em sistema de produção orgânica é acompanhada de alterações relacionadas à intensidade e à diversidade de infestação/infecção dos animais por ecto e endoparasitos, tornando indispensável o emprego de medidas profiláticas (Almeida, 2013).

\section{Avicultura orgânica}

No protocolo de criação orgânica de aves, um dos grandes objetivos é a questão de bem-estar dos animais que serão abatidos e das pessoas que realizarão o trabalho de cuidar desses animais e zelar por eles (Arenales et al., 2008). A produção orgânica possui algumas particularidades com relação aos outros sistemas de criação convencionais e caipiras, além de possuir a certificação orgânica, não e permitido a utilização de agrotóxicos, adubos químicos sintéticos e tem como objetivo aumentar a biodiversidade e os ciclos biológicos, atingindo melhor os sistemas naturais visando à sustentabilidade. O tempo de criação é mais longo quando comparado ao convencional (Matt et al., 2011). A produção de alimentos, que buscou fontes sustentáveis de produção, utilizando tecnologia limpa como a agricultura orgânica, tem cada vez mais, ganhando impulso em todos os continentes, movimentando o mercado internacional, ampliando os investimentos daqueles que se dedicam à agricultura sustentável (Camargo Filho et al., 2004).

A garantia da qualidade dos produtos orgânicos no Brasil é controlada de três maneiras que serão descritas no tópico legislação e certificação de Produtos orgânicos no Brasil, esses elos são: Certificação, o Controle Social para Venda Direta sem Certificação e os Sistemas participativos de Garantia, formando o Sistema Brasileiro de Avaliação da Conformidade Orgânica (SisOrg) (Portal agricultura, 2014).

Para melhor compreensão do sistema de cultivo orgânico e das práticas que devem ser adotadas como propriedade, é necessário recorrer as Normativas $\mathrm{n}^{\circ} 64$ de dezembro de 2008 , a qual regulamenta e estabelece normas técnicas para os Sistemas Orgânicos de Produção Animal e Vegetal no Brasil, baseada na lei 10.831 de 23 de dezembro de 2003 (Brasil, 2003), esta última ressalta no Art. $1^{\circ}$ que "considera-se sistema orgânico de produção agropecuária todo aquele em que se adotam técnicas específicas, mediante a otimização do uso dos recursos naturais e socioeconômicos disponíveis e o respeito à integridade cultural das comunidades rurais, tendo por objetivo a sustentabilidade econômica e ecológica, a maximização dos benefícios sociais, à minimização da dependência de energia não renovável, empregando, sempre que possíveis métodos culturais biológicos e mecânicos, em contraposição ao uso de materiais sintéticos, a eliminação do uso de organismos geneticamente modificados e radiações ionizantes, em qualquer fase do processo de produção, processamento, armazenamento, distribuição e comercialização, e a proteção do meio ambiente" (Zibtte, et al., 2011).

Todo o sistema de criação dever ser ao ar livre, ou seja, o confinamento é condenável. Nas primeiras semanas, as aves devem ficar continuamente abrigadas, e, na fase final, livres durante o dia e, à noite, recolhidas em galpões amplos, com acesso a poleiros que devem ser fechados para evitar a ação de predadores. Estes animais devem ser criados em piquetes com acesso a pastagem, onde vão realizar suas funções inerentes à espécie de exploração do meio ambiente, repouso de acordo com as características da espécie. Esta dinâmica minimiza o estresse e, dessa forma, a competição entre as aves diminui efetivamente (Arenales et al., 2008). Estudos mostram que poedeiras criadas no sistema orgânico produzem ovos com melhor qualidade interna que ovos produzidos por poedeiras criadas no sistema convencional em gaiolas, pois as aves criadas nesses sistemas, possuem menor demanda de intensidade de postura, em comparação às altas produtividades alcançadas por aves criadas no sistema convencional de gaiolas (Van et al., 2004; Castellini et al., 2006; Duric-Stojcic et al., 2009).

Levantamentos realizados pelo Mapa através do Cadastro Nacional dos Produtores Orgânicos, números de avaliadores do setor tem dobrado desde o ano de 2013, esse mesmo quantitativo no período de um ano (2014 á 2015) teve um crescimento de $51,70 \%$, ou seja, cresceu de 6.719 (2014) para 10.194 (2015). Nos anos anteriores o país contava com 79 Organizações de Controle Social (OCSs) e 4 Organismos Participativos de Avaliação da Conformidade (OPACs).Números que em 2011 subiram para 163 e 11 respectivamente. O mercado orgânico nas últimas décadas apresentou crescimento entre 40 e $50 \%$ no período da virada do milênio, isto 
em termos de volumes comercializados. A maior parte da produção orgânica no Brasil está concentrada nas regiões Sul e Sudeste. Cerca de $85 \%$ da produção orgânica brasileira é exportada, principalmente para a Europa, Estados Unidos e Japão, os outros $15 \%$ são comercializados no mercado interno (Arenales et al., 2008).

Algo que precisa ser trabalhando quando falamos de sistema de produção orgânico é a ideia que seja um sistema em que não existe a preocupação com a sanidade, com alimentação de qualidade e que atenda às exigências nutricionais dos animais criados nesse sistema. Uma pesquisa feita recentemente mostra que a maioria dos consumidores não sabe diferenciar um produto de origem caipira de um orgânico.

\section{Certificação de produtos orgânicos}

Os produtos considerados orgânicos são produzidos em um ambiente com base do processo produtivo os princípios agroecológicos que contemplam o uso responsável do solo, água, ar, e dos demais recursos naturais, respeitando as relações sociais e culturais (MAPA, 2015). Segundo o Mapa, na agricultura orgânica não é permitido o uso de substâncias que coloquem em risco a saúde humana e o meio ambiente. Como também a utilização de fertilizantes sintéticos, solúveis, agrotóxicos, e transgênicos. O Brasil, em função de possuir diferentes tipos de solo, clima, uma biodiversidade aliada a uma grande diversidade cultural, é sem dúvida um dos países com maior potencial para o crescimento da produção orgânica.

Diante disso, para os produtores que gostariam de comercializar seus produtos como orgânicos é necessário a certificação pelo MAPA, existem duas formas para a regularização, pode se obter a certificação por meio de um Organismo de Avaliação da Conformidade Orgânica (OAC) credenciada junto ao Ministério da Agricultura, Pecuária e AbastecimentoMAPA, ou ainda pode se organizar em grupo e se cadastrar junto ao MAPA para realizar a venda direta sem certificação.

Nesta última certificação o produtor não poderá escoar a produção para terceiros, somente em feiras livres (direto ao consumidor) e para as compras do governo (merenda escolar e CONAB). Quando o produto é certificado a venda pode ocorrer para indústrias, supermercados, restaurante, hotéis, internet, entre outros meios. Ressaltando que na venda em feira livre (sem a Certificação) o produtor deverá apresentar um documento denominado de Declaração de Cadastro, onde consta que ele esta cadastrado ao Ministério da Agricultura Pecuária Abastecimento (MAPA) e que faz parte de um grupo que se responsabiliza por ele. Nesse caso, só o produtor, alguém de sua família ou se seu grupo pode estar na barraca, vendendo o produto. Essa declaração deve ser mostrada sempre que o consumidor e a fiscalização solicitarem (MAPA, 2015).

\section{Aquisição dos animais}

$\mathrm{O}$ fator aquisição dos animais destinados à criação orgânica, assim como ocorre em qualquer sistema de produção, deve-se escolher animais que sejam resistentes a endo e ectoparasitas; isto pode ser potencializado quando o animal recebe todas as condições necessárias para atender suas necessidades, como uma alimentação adequada que atenda suas exigências nutricionais, um ambiente que permita a ele expressar o seu comportamento normal, ou seja, livre de sofrer estresse durante todas as fases de produção (Almeida, 2013).

As aves podem ser adquiridas com até 16 semanas de idade, já vacinadas contra as doenças obrigatórias e as de ocorrência local (como por exemplo, Marek, coccidiose, bouba, bronquite, doença de newcastle, gumboro, paratifo aviário) (Figueiredo \& Soares, 2012).

Pesquisas recentes na área de melhoramento genético foram realizadas pela Embrapa Suínos e Aves com o objetivo de produzir fenótipos mais adaptados à produção colonial/Orgânica/ agro ecológica de aves para corte e postura. As linhagens Embrapa 041 (corte) e Embrapa 031 e 051 (postura) foram comparadas a outras linhagens existentes no mercado. Os resultados de desempenho dos frangos da linhagem Ross e Embrapa 041, este último se mostra satisfatório aos 84 dias. Todas as aves atingiram a idade de abate aos 70 dias, seja em sistema confinado ou semi-confinado (Avila et al., 2006). Já á linhagem Label Rouge precisou de quase 90 dias para atingir seu potencial máximo de produção (Schmidt et al., 2005).

As linhagens Embrapa 051 e 031 tiveram uma produção significativa quando alojadas em sistema confinado e semi-confinado, ambas as linhagens apresentam produção equilibrada, ressaltando ainda que a linhagem 051 obteve uma produção perto do seu nível máximo de produção 
(255 ovos) e a linhagem Embrapa 031 que produziu cerca de 248 ovos, aproximando-se do seu potencial genético (248 ovos) no mesmo período (Avila et al., 2006).

\section{Alimentação}

Em toda a fase de criação não é permitido o fornecimento de ração comercial aos animais criados nesse sistema de produção, sendo assim, toda fonte de alimento deverá ser de origem orgânica. E se possível, que seja elaborada na própria propriedade, além de ocasionar uma diminuição nos custos com a nutrição, ainda pode-se fornecer alimentos alternativos, como adição de frutas, vegetais, verduras e raízes (Arenales et al.,, 2008). Condição essencial para produzir uma ração orgânica balanceada para galinhas de postura é que a propriedade rural certificada, ou o conjunto de propriedades certificadas ou acreditadas produzam os ingredientes orgânicos (Ludke et al., 2010).

A água também é fundamental para os processos vitais do organismo, como a digestão, absorção dos alimentos e eliminação das secreções. Sendo que deve ser fornecida em abundância aos animais (Arenales et al., 2008). Devemos nos atentar no momento da produção dos alimentos que vão compor a ração quanto ao nível de produtividade, qualidade de cada cultura, as exigências nutricionais das aves por fase de produção e o conhecimento da composição nutricional dos ingredientes que se deseja cultivar determinam o adequado planejamento do sistema orgânico (Ludke et al., 2010).

A ração das aves deve ser balanceada em energia metabolizável, proteína bruta, cálcio e fósforo para as fases de pré-postura (15 a 18 semanas), postura I (19 a 40 semanas) e postura II (acima de 41 semanas), a mesma deverá ser preparada com ingredientes cultivados na propriedade e oferecida no comedouro na quantidade necessária para atender às exigências das aves em cada fase (Figueiredo \& Soares, 2012).

\section{Sanidade}

Em sistemas orgânicos utilizam-se os fitoterápicos como forma de prevenção e tratamento de possíveis enfermidades que possam ocorrer dentro do sistema de produção. Recomenda-se que em todo sistema respeite os recursos naturais e tenha como objetivo tornar-se auto-sustentável, visando preservar a biodiversidade dos ecossistemas (Arenales et al., 2008).

Seguindo as normas da regulamentação a Produção Animal Orgânico (Brasil, 2003; REPAB, 2007), devem ser empregadas as terapêuticas homeopática, fitoterápica e a acupuntura, sendo restrito o uso de produtos químico de síntese. A fitoterapia é a utilização de plantas com propriedades medicinais, usadas como terapêuticas através dos recursos presentes no ambiente em que a produção está inserida, podemos destacar o uso do alho, semente de abóbora, o tronco da bananeira como princípios ativos da verminose, ressaltando que o alho é um imuno e estimulante do sistema imunitário (Arenales et al., 2008).

No mercado, existem fitoterápicos e homeopáticos que podem ser utilizados durante $o$ manejo profilático da criação orgânica, deve-se ter atenção no momento da compra desses produtos, pois esses devem ser registrados pelo Ministério da Agricultura, Pecuária e Abastecimento-MAPA (Almeida, 2013).

Relacionado ao controle sanitário da produção orgânica, existe o uso indiscriminado de drogas anti-helmínticas que pode ocasionar na seleção de nematóides resistentes ou de nematóides que desenvolvem resistência aos anti-helmínticos. Portanto, o uso de plantas medicinais com essa função surge como uma possibilidade de tratamento simples e barato, resgatando a cultura da medicina popular (Soares et al., 2011). Ressaltando assim, a deficiência dos estudos científicos relacionados a essa forma de manejo profilático no sistema orgânico de produção animal.

\section{Conclusão}

A criação orgânica é um dos sistemas de criação para aves de postura que podemos obter uma produção satisfatória atendendo também às exigências de um mercado consumidor cada vez mais atento com relação à origem dos alimentos e condições em que os animais destinados ao consumo humano são criados.

Diversos trabalhos ressaltam que é possível produzir e garantir alimento de qualidade, com a utilização de recursos naturais disponíveis na propriedade. E que a certificação dos produtos orgânicos garante a autenticidade e segurança de que o alimento foi produzido em um sistema que segue as normas de criação orgânica. 
O principal fator desse modelo de sistema alternativo que possibilita um excelente retorno econômico é que o mesmo atende as requisições de um dos principais mercados consumidores do mundo, a União Europeia, cumprindo também os preceitos de bem estar animal. Este é um dos temas mais debatidos da atualidade, possibilitando que os animais possam expressar todo o seu comportamento natural, livre de fome e sede, sendo esta uma das cinco liberdades que compõem as normas de bem estar animal.

Ainda necessita de pesquisas mais avançadas na área, principalmente no diz respeito ao manejo profilático, uma vez que na produção orgânica são utilizados conhecimentos populares para enfermidades que venham à produção. Necessita-se também de pesquisas voltadas para o domínio da forma de criação, ressaltando que esses resultados no sistema convencional os lucros obtidos surgem em um menor espaço de tempo quando comparado ao sistema de criação orgânico, que demanda um tempo maior para a obtenção desses mesmos resultados. Lembrando que os produtores estão acostumados a terem esses lucros com mais rapidez nos sistemas de criação convencional.

\section{Referências Bibliográficas}

Almeida. L. R. (2013). Manejo de Parasitoses em Sistema Orgânico de Produção de Leite. Arquivos do Instituto Biológico. 80,129-134.

Arenales, M.D.C., Rossi.F., Ferreira.R.G.S., Ferreira.D.G.S (2008). Criação orgânica de frangos de corte e aves de postura. Editora Aprenda fácil. Viçosa-MG.

Alvarado Hullanco. M.B. (2004). Aplicação de um sistema de classificação de carcaças e cortes e efeito após abate da qualidade de cortes de frangos criados em sistema alternativo. Escola Superior Agrícola Luiz de Queiroz da Universidade de São Paulo.

Avila, V. S., Coldebela, A., Brum, P. A. R., Schmidt, G. S., Lima, G. J. M. \& Figueiredo, E. A. P. (2006). Efeito de Duas Densidades de Alojamento da Poedeira Embrapa 031 Criada em piso Sobre Cama. In: Reunião Anual da Sociedade Brasileira de Zootecnia.

Brasil. (2003). Lei $n^{\circ} 10.831$ de 23 de dezembro 2003. Dispõe sobre o sistema orgânico de produção agropecuária. Diário Oficial da
República Federativa do Brasil, Brasília. Seção 1, pág.8.

Camargo Filho, W.P.d, Camargo, F.P.d, Camargo, A.M.M.P.d, Aleves, H.S. (2004). Algumas considerações sobre a construção da cadeia de produtos orgânicos. Informações econômicas, 34, 2.

Castellini, C., Perella, F. \& Mugnai. (2006). Welfare, productivity and qualitative traits of egg in laying hens reared under different rearing systems. In: XII European Poultry Conference, Anais... Verona, EUA. P 10-14.

Duric-Stojcic, M., Peric, L. \& Bjedov, S. (2009). Milosevic, $N$. the quality of table eggs produced by different housing systems. Biotechnology in Animal Husbandry, 25, 1103-1108.

Figueiredo, E. A. P. D. \& Soares, J. P. G. (2012). Sistemas orgânicos de produção animal: dimensões. técnicas e econômicas. Anais da 49 ${ }^{\mathrm{a}}$. Reunião Anual da Sociedade Brasileira de Zootecnia. A Produção Animal no Mundo em Transformação.

Furlan, R.L., Macari, M., Mateus, J.R. (2006). Bem estar das aves e sua implicações sobre o desenvolvimento e produção. Disponível em: <www.engomix.com/bem_estar_das_aves_p_ artigos_17_AVG_htm>. Acesso em 26/02/2016.

Khatounian, C.A. (2011). A Reconstrução Ecológica da Agricultura. Botucatu, ISBN 85-88581-26-4.

Ludke, V., Figueiredo, E. A. P., Avila, V.S.d. \& Mazzuco. H. (2010). Alimentos e Alimentação de Galinhas Poedeiras em Sistemas Orgânicos de Produção. Embrapa Suínos e Aves. Concórdia, Santa Catarina.

Mapa (2015)- Ministério da Agricultura. Lei $\mathrm{N}^{\circ}$ 10.831, (2003). Dispõe sobre agricultura Orgânica é dá outras Providências. Disponível em www.agricultura.gov.br.

Mapa (1999). Ministério da Agricultura, Pecuária e Abastecimento. Ofício circular DOI/DIPOA no $\mathrm{N}^{\circ} 60 / 99$ de 04/11/99. 1999b. 2 p. Disponível em: www.agricultura.gov.br.

MAPA (2015)- Ministério da Agricultura. Regularização da produção Orgânica. Disponível em www.agricultura.gov.br.

Matt, D., Rembialkowska, E., Luik, A., Pehme, S. \& Peetsmann, E. (2011). Quality of organic 
vs. Conventional food and effects on health. Estonian University of Sciences, Tartu, Estonia.

Menezes, P.C, Cavalcante, V.F.T, Lima E.R, Neto J.E. (2009) Aspectos produtivos e econômicos de poedeiras comerciais submetidas a diferentes densidades de alojamentos. Revista Brasileira de Zootecnia., 38, 2224-2229.

Pasian, I.M. Gamaero, A.H. (2007). Mercado Para a Criação de Poedeiras em Sistemas do Tipo Orgânico, Caipira e Convencional- XLV Congresso do SOBER- Sociedade Brasileira de Economia, Administração e Sociologia Rural, 22 a 25 de julho,UEL Londrina.

Pasian, I.M.D.L. (2006). Bem estar em aves poedeiras. Trabalho realizado na disciplina de comportamento e bem estar animal, Pirassununga. Faculdade de Medicina Veterinária e Zootecnia, Universidade de São Paulo.

Portal agricultura (2014). Notícias, Assessoria de Comunicação Social. Luana Brasil. Ano 2014. Disponível em: www.agricultura.gov.br. Acesso em 03/02/15.

REPAB (2007). Réglement Européendes Productions Animales Biologiques. Cahier des Charges concernant le mode de production Biologique des Animaux. Direction des politiques économiques et internationale, Ministère de l'Agriculture et la Pêche, France.

Schmidt, G. S, Figueiredo, E. A. P, Lima, G. J. M. M. \& Avila, V.S. (2005). Alternativas de dimensionamento e organização para a produção de frango de corte na agricultura
Familiar. Concórdia: Embrapa Suínos e Aves, (Comunicado Técnico, 396). 11p.

Soares, J.P.G, Aroeira, L.J.M, Fonseca, A.H.F, Fagundes, G.M, Silva, J.B. ( 2011). Produção orgânica de leite: Desafios e perspectivas. In: Marcondes, M.I et al.,(org.). Anais do III Simpósio Nacional de bovinocultura leiteira e I simpósio internacional de bovinocultura leiteira. 1 ed. Viçosa: Suprema Gráfica e Editora, V.1. p.13-43.

União Brasileira de Avicultura, Relatório Anual (2013). Disponível em www.ubabef.com.br.

Van, D. B. H., Paramenter, H.K. \& Kem, B. (2004). Effects of housing system (outdoor vs cages) and age of laying hens on egg characteristics. British Poultry Science, 45,745-752.

Zabaleta, J. P. (2013) Avicultura Colonial., Embrapa. Disponível em www.cpact.embrapa.br.

Zibtte, A.P., Corrêia, F.A., Signor, A.A. ( 2011). Livro Produção Orgânica Animal. Cap,1. Agropecuária Orgânica como Alternativa de Desenvolvimento Sustentavel. Pag, 3-11.

Recebido em Outubro 2, 2015

Aceito em Dezembro 2, 2015

License information: This is an open-access article distributed under the terms of the Creative Commons Attribution License, which permits unrestricted use, distribution, and reproduction in any medium, provided the original work is properly cited. 